This is an Accepted Manuscript of an article published by European Mathematical Society in "Journal of the European Mathematical Society" 2016, available online:

http://www.ems-ph.org/journals/show_abstract.php?issn=1435-9855\&vol=18\&iss=12\&rank=9 
Xavier Cabré · Xavier Ros-Oton · Joaquim Serra

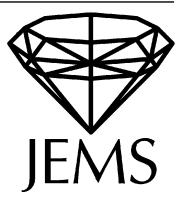

\title{
Sharp isoperimetric inequalities via the ABP method
}

Received January 8, 2014 and in revised form May 21, 2015

\begin{abstract}
Given an arbitrary convex cone of $\mathbb{R}^{n}$, we find a geometric class of homogeneous weights for which balls centered at the origin and intersected with the cone are minimizers of the weighted isoperimetric problem in the convex cone. This leads to isoperimetric inequalities with the optimal constant that were unknown even for a sector of the plane. Our result applies to all nonnegative homogeneous weights in $\mathbb{R}^{n}$ satisfying a concavity condition in the cone. The condition is equivalent to a natural curvature-dimension bound and also to the nonnegativity of a Bakry-Émery Ricci tensor. Even though our weights are nonradial, balls are still minimizers of the weighted isoperimetric problem. A particular important case is that of monomial weights. Our proof uses the ABP method applied to an appropriate linear Neumann problem.

We also study the anisotropic isoperimetric problem in convex cones for the same class of weights. We prove that the Wulff shape (intersected with the cone) minimizes the anisotropic weighted perimeter under the weighted volume constraint.

As a particular case of our results, we give new proofs of two classical results: the Wulff inequality and the isoperimetric inequality in convex cones of Lions and Pacella.
\end{abstract}

Keywords. Isoperimetric inequalities, densities, convex cones, homogeneous weights, Wulff shapes, ABP method

\section{Introduction and results}

In this paper we study isoperimetric problems with weights, also called densities. Given a weight $w$ (that is, a positive function $w$ ), one wants to characterize minimizers of the weighted perimeter $\int_{\partial E} w$ among those sets $E$ having weighted volume $\int_{E} w$ equal to a given constant. A set solving the problem, if it exists, is called an isoperimetric set or simply a minimizer. This question, and the associated isoperimetric inequalities with weights, have attracted much attention recently: see for example [43], [36], [17], [23], and [41].

X. Cabré: Departament de Matemàtiques, Universitat Politècnica de Catalunya, Diagonal 647, 08028 Barcelona, Spain, and ICREA, Pg. Lluis Companys 23, 08010 Barcelona, Spain;

e-mail: xavier.cabre@upc.edu

X. Ros-Oton: Department of Mathematics, The University of Texas at Austin, 2515 Speedway, Austin, TX 78751, USA; e-mail: ros.oton@math.utexas.edu

J. Serra: Department of Mathematics, ETH Zürich, Raemistrasse 101, 8092 Zürich, Switzerland; e-mail: joaquim.serra@math.ethz.ch

Mathematics Subject Classification (2010): Primary 28A75; Secondary 35A23, 49Q20 
The solution to the isoperimetric problem in $\mathbb{R}^{n}$ with a weight $w$ is known only for very few weights, even in the case $n=2$. For example, in $\mathbb{R}^{n}$ with the Gaussian weight $w(x)=e^{-|x|^{2}}$ all the minimizers are half-spaces [3, 16], and with $w(x)=e^{|x|^{2}}$ all the minimizers are balls centered at the origin [46]. Instead, mixed Euclidean-Gaussian densities lead to minimizers that have a more intricate structure of revolution [26]. The radial homogeneous weight $|x|^{\alpha}$ has been considered very recently. In the plane $(n=2)$, minimizers for this homogeneous weight depend on the values of $\alpha$. On the one hand, CarrollJacob-Quinn-Walters [13] showed that when $\alpha<-2$ all minimizers are $\mathbb{R}^{2} \backslash B_{r}(0)$, $r>0$, and that when $-2 \leq \alpha<0$ minimizers do not exist. On the other hand, when $\alpha>0$ Dahlberg-Dubbs-Newkirk-Tran [19] proved that all minimizers are circles passing through the origin (in particular, not centered at the origin). Note that this result shows that even radial homogeneous weights may lead to nonradial minimizers. However, if the weight is radial and log-convex, G. Chambers [14] has established that balls in $\mathbb{R}^{n}$ centered at the origin are isoperimetric regions for any given volume. This was known as the Log-Convex Density Conjecture; see the blog of Frank Morgan [42].

Weighted isoperimetric inequalities in cones have also been considered. In these results, the perimeter of $E$ is taken relative to the cone, that is, not counting the part of $\partial E$ that lies on the boundary of the cone. In [20] Díaz-Harman-Howe-Thompson consider again the radial homogeneous weight $w(x)=|x|^{\alpha}$, with $\alpha>0$, but now in an open convex cone $\Sigma$ of angle $\beta$ in the plane $\mathbb{R}^{2}$. Among other things, they prove that there exists $\beta_{0} \in(0, \pi)$ such that for $\beta<\beta_{0}$ all minimizers are $B_{r}(0) \cap \Sigma, r>0$, while these circular sets about the origin are not minimizers for $\beta>\beta_{0}$.

Also related to the weighted isoperimetric problem in cones is a recent result by Brock-Chiaccio-Mercaldo [4]. Assume that $\Sigma$ is any cone in $\mathbb{R}^{n}$ with vertex at the origin, and consider the isoperimetric problem in $\Sigma$ with any weight $w$. Then, for $B_{R}(0) \cap \Sigma$ to be an isoperimetric set for every $R>0$, a necessary condition is that $w$ admits the factorization

$$
w(x)=A(r) B(\Theta),
$$

where $r=|x|$ and $\Theta=x / r$. Our main result-Theorem 1.3 below-gives a sufficient condition on $B(\Theta)$ whenever $\Sigma$ is convex and $A(r)=r^{\alpha}, \alpha \geq 0$, to guarantee that $B_{R}(0) \cap \Sigma$ are isoperimetric sets.

Our result states that Euclidean balls centered at the origin solve the isoperimetric problem in any open convex cone $\Sigma$ of $\mathbb{R}^{n}$ (with vertex at the origin) for a certain class of nonradial weights. More precisely, our result applies to all nonnegative continuous weights $w$ which are positively homogeneous of degree $\alpha \geq 0$ and such that $w^{1 / \alpha}$ is concave in the cone $\Sigma$ in case $\alpha>0$. That is, using the previous notation, $w=r^{\alpha} B(\Theta)$ must be continuous in $\bar{\Sigma}$ and $r B^{1 / \alpha}(\Theta)$ must be concave in $\Sigma$. This condition is equivalent to a natural curvature-dimension bound and also to the nonnegativity of a Bakry-Émery Ricci tensor (see Remark 1.4). Our result establishes weighted isoperimetric inequalities with an optimal constant that were unknown even for a sector of the plane. Note that even though our weights are nonradial, still balls are minimizers of the weighted isoperimetric problem. As we will see, a particular important case is that of monomial weights. Our results were announced in the note [10]. 
We also solve weighted anisotropic isoperimetric problems in cones for the same class of weights. In these problems, the perimeter of a domain $\Omega$ is given by

$$
\int_{\partial \Omega \cap \Sigma} H(v(x)) w(x) d S
$$

where $v(x)$ is the unit outward normal to $\partial \Omega$ at $x$, and $H$ is a positive convex function, positively homogeneous of degree one.

In the isotropic case, taking the first variation of weighted perimeter (see [46]), one sees that the (generalized) mean curvature of $\partial \Omega$ with the density $w$ is

$$
H_{w}=H_{\mathrm{eucl}}+\frac{1}{n} \frac{\partial_{\nu} w}{w}
$$

where $v$ is is the unit outward normal to $\partial \Omega$ and $H_{\text {eucl }}$ is the Euclidean mean curvature of $\partial \Omega$. It follows that balls centered at the origin intersected with the cone have constant mean curvature whenever the weight is of the form (1.1). However, as we have seen in several examples presented above, it is far from being true that the solution of the isoperimetric problem for all the weights satisfying (1.1) are balls centered at the origin intersected with the cone. Our result provides a large class of nonradial weights for which, remarkably, Euclidean balls centered at the origin (intersected with the cone) solve the isoperimetric problem.

In our isoperimetric inequality with a homogeneous weight $w$ of degree $\alpha$ in a convex cone $\Sigma \subset \mathbb{R}^{n}$, balls intersected with the cone are minimizers. As a consequence, this yields the following Sobolev inequality in which we can compute its optimal constant (as well as the shape of the extremizers). If $D=n+\alpha, 1 \leq p<D$, and $p_{*}=p D /(D-p)$, then

$$
\left(\int_{\Sigma}|u|^{p_{*}} w(x) d x\right)^{1 / p_{*}} \leq C_{w, p, n}\left(\int_{\Sigma}|\nabla u|^{p} w(x) d x\right)^{1 / p}
$$

for all smooth functions $u$ with compact support in $\mathbb{R}^{n}$-in particular, not necessarily vanishing on $\partial \Sigma$. This is a consequence of our isoperimetric inequality (Theorem 1.3) and a weighted radial rearrangement of Talenti [47], since these two results yield the radial symmetry of extremizers.

When the homogeneous weight is a monomial

$$
w(x)=x_{1}^{A_{1}} \cdots x_{n}^{A_{n}} \quad \text { in } \Sigma=\left\{x \in \mathbb{R}^{n}: x_{i}>0 \text { whenever } A_{i}>0\right\}
$$

(here $A_{i} \geq 0$ ), this type of Sobolev inequalities appeared naturally in our recent papers $[8,9]$ on reaction-diffusion problems in domains with symmetry of double revolution. These weighted Sobolev inequalities have interesting applications to the regularity of local minimizers in such domains.

In Section 2 we give a list of weights $w$ to which our result applies. Some concrete examples are (1.4),

$$
w(x)=\operatorname{dist}(x, \partial \Sigma)^{\alpha} \quad \text { in } \Sigma \subset \mathbb{R}^{n}
$$

where $\Sigma$ is any open convex cone and $\alpha \geq 0$ (see example (ii) in Section 2), and

$$
x^{a} y^{b} z^{c}, \quad\left(a x^{r}+b y^{r}+c z^{r}\right)^{\alpha / r}, \quad \text { or } \frac{x y z}{x y+y z+z x} \quad \text { in } \Sigma=(0, \infty)^{3},
$$


where $a, b, c$ are nonnegative numbers, $r \in(0,1]$ or $r<0$, and $\alpha>0$ (see examples (iv), (v), and (vii), respectively).

The proof of our main result follows the ideas introduced by the first author [6, 7] in a new proof of the classical isoperimetric inequality (the classical inequality corresponds to the weight $w \equiv 1$ and the cone $\Sigma=\mathbb{R}^{n}$ ). Our proof consists in applying the ABP method to an appropriate linear Neumann problem involving the operator

$$
w^{-1} \operatorname{div}(w \nabla u)=\Delta u+\frac{\nabla w}{w} \cdot \nabla u,
$$

where $w$ is the weight.

The Alexandroff-Bakelman-Pucci (ABP) method is a classical tool in the theory of second order elliptic equations of nondivergence form. This method was used by those authors in the sixties to establish an $L^{\infty}$ bound for solutions to equations with bounded measurable coefficients. In Section 3 we explain in more detail the method and its relation to the proof of our main result.

\subsection{The setting}

The classical isoperimetric problem in convex cones was solved by P.-L. Lions and F. Pacella [33] in 1990. Their result states that among all sets $E$ with fixed volume inside an open convex cone $\Sigma$, the balls centered at the vertex of the cone minimize the perimeter relative to the cone (recall that the part of the boundary of $E$ that lies on the boundary of the cone is not counted).

Throughout the paper, $\Sigma$ is an open convex cone in $\mathbb{R}^{n}$. Recall that given a measurable set $E \subset \mathbb{R}^{n}$, the relative perimeter of $E$ in $\Sigma$ is defined by

$$
P(E ; \Sigma):=\sup \left\{\int_{E} \operatorname{div} \sigma d x: \sigma \in C_{c}^{1}\left(\Sigma, \mathbb{R}^{n}\right),|\sigma| \leq 1\right\} .
$$

When $E$ is smooth enough,

$$
P(E ; \Sigma)=\int_{\partial E \cap \Sigma} d S .
$$

The isoperimetric inequality in cones of Lions and Pacella reads as follows.

Theorem 1.1 ([33]). Let $\Sigma$ be an open convex cone in $\mathbb{R}^{n}$ with vertex at 0 , and $B_{1}:=$ $B_{1}(0)$. Then

$$
\frac{P(E ; \Sigma)}{|E \cap \Sigma|^{(n-1) / n}} \geq \frac{P\left(B_{1} ; \Sigma\right)}{\left|B_{1} \cap \Sigma\right|^{(n-1) / n}}
$$

for every measurable set $E \subset \mathbb{R}^{n}$ with $|E \cap \Sigma|<\infty$.

The assumption of convexity of the cone cannot be removed. In the same paper [33] the authors give simple examples of nonconvex cones for which inequality (1.5) does not hold. The idea is that for cones having two disconnected components, (1.5) is false since it requires less perimeter to concentrate all the volume in one of the two subcones. A connected (but nonconvex) counterexample is then obtained by joining the two components by a conic open thin set. 
The proof of Theorem 1.1 given in [33] is based on the Brunn-Minkowski inequality

$$
|A+B|^{1 / n} \geq|A|^{1 / n}+|B|^{1 / n},
$$

valid for all nonempty measurable sets $A$ and $B$ of $\mathbb{R}^{n}$ for which $A+B$ is also measurable; see [29] for more information on this inequality. As a particular case of our main result, in this paper we provide a totally different proof of Theorem 1.1. This new proof is based on the ABP method.

Theorem 1.1 can be deduced from a degenerate case of the classical Wulff inequality stated in Theorem 1.2 below. This is because the convex set $B_{1} \cap \Sigma$ is the Wulff shape (1.8) associated to some appropriate anisotropic perimeter. As explained below in Section 3, this idea is crucial in the proof of our main result. This fact has also been used recently by Figalli and Indrei [22] to prove a quantitative isoperimetric inequality in convex cones. From it, one deduces that balls centered at the origin are the unique minimizers in (1.5) up to translations that leave invariant the cone (if they exist). This had been established in [33] in the particular case when $\partial \Sigma \backslash\{0\}$ is smooth (and later in [45], which also classified stable hypersurfaces in smooth cones).

The following is the notion of anisotropic perimeter. We say that a function $H$ defined in $\mathbb{R}^{n}$ is a gauge when

$H$ is nonnegative, positively homogeneous of degree one, and convex.

Somewhere in the paper we may require a function to be homogeneous; by this we always mean positively homogeneous.

Any norm is a gauge, but a gauge may vanish on some unit vectors. We need to allow this case since it will occur in our new proof of Theorem 1.1 - which builds from the cone $\Sigma$ a gauge that is not a norm.

For a smooth set $E \subset \mathbb{R}^{n}$, the anisotropic perimeter associated to the gauge $H$ is given by

$$
P_{H}(E)=\int_{\partial E} H(v(x)) d S,
$$

where $v(x)$ is the unit outward normal at $x \in \partial E$.

The Wulff shape associated to $H$ is defined as

$$
W=\left\{x \in \mathbb{R}^{n}: x \cdot v<H(v) \text { for all } v \in \mathbb{S}^{n-1}\right\} .
$$

We will always assume that $W \neq \emptyset$. Note that $W$ is an open set with $0 \in \bar{W}$. To visualize $W$, it is useful to note that it is the intersection of the half-spaces $\{x \cdot v<H(v)\}$ over all $v \in \mathbb{S}^{n-1}$. In particular, $W$ is a convex set.

For general measurable sets $E \subset \mathbb{R}^{n}$, the anisotropic perimeter is defined as

$$
P_{H}(E):=\sup \left\{\int_{E} \operatorname{div} \sigma d x: \sigma \in C_{c}^{1}\left(\mathbb{R}^{n}, \mathbb{R}^{n}\right), \sigma(x) \in W \text { for } x \in \mathbb{R}^{n}\right\} .
$$

When $E$ is smooth enough, this definition coincides with (1.7). 
From the definition (1.8) of the Wulff shape it follows that, given an open convex set $W \subset \mathbb{R}^{n}$ with $0 \in \bar{W}$, there is a unique gauge $H$ such that $W$ is the Wulff shape associated to $H$. Indeed, $H$ is uniquely defined by

$$
H(x)=\inf \left\{t \in \mathbb{R}: W \subset\left\{z \in \mathbb{R}^{n}: z \cdot x<t\right\}\right\} .
$$

Note that, for each direction $v \in \mathbb{S}^{n-1},\{x \cdot v=H(v)\}$ is a supporting hyperplane of $W$. Thus, for almost every point $x$ on $\partial W$-those for which the outer normal $v(x)$ exists-we have

$$
x \cdot v(x)=H(v(x)) \quad \text { a.e. on } \partial W .
$$

Note also that since $W$ is convex, it is a Lipschitz domain. Hence, we can use the divergence theorem to obtain the formula

$$
P_{H}(W)=\int_{\partial W} H(v(x)) d S=\int_{\partial W} x \cdot v(x) d S=\int_{W} \operatorname{div}(x) d x=n|W|,
$$

relating the volume and the anisotropic perimeter of $W$.

When $H$ is positive on $\mathbb{S}^{n-1}$, it is natural to introduce its dual gauge $H^{\circ}$, defined by

$$
H^{\circ}(z)=\sup _{H(y) \leq 1} z \cdot y
$$

Then the last condition on $\sigma$ in the definition of $P_{H}(\cdot)$ is equivalent to $H^{\circ}(\sigma) \leq 1$ in $\mathbb{R}^{n}$, and the Wulff shape can be written as $W=\left\{H^{\circ}<1\right\}$.

Some typical examples of gauges are

$$
H(x)=\|x\|_{p}=\left(\left|x_{1}\right|^{p}+\cdots+\left|x_{n}\right|^{p}\right)^{1 / p}, \quad 1 \leq p \leq \infty .
$$

Then $W=\left\{x \in \mathbb{R}^{n}:\|x\|_{p^{\prime}}<1\right\}$, where $p^{\prime}$ is such that $1 / p+1 / p^{\prime}=1$.

The following is the celebrated Wulff inequality.

Theorem 1.2 ([53, 48, 49]). Let $H$ be a gauge in $\mathbb{R}^{n}$ which is positive on $\mathbb{S}^{n-1}$, and let $W$ be its associated Wulff shape. Then, for every measurable set $E \subset \mathbb{R}^{n}$ with $|E|<\infty$,

$$
\frac{P_{H}(E)}{|E|^{(n-1) / n}} \geq \frac{P_{H}(W)}{|W|^{(n-1) / n}} .
$$

Moreover, equality holds if and only if $E=a W+b$ for some $a>0$ and $b \in \mathbb{R}^{n}$, up to $a$ set of measure zero.

This result was first stated without proof by Wulff [53] in 1901. His work was followed by Dinghas [21], who studied the problem within the class of convex polyhedra. He used the Brunn-Minkowski inequality. Some years later, Taylor [48, 49] finally proved Theorem 1.2 for sets of finite perimeter; see $[50,25,38]$ for more information on this topic. As a particular case of our technique, in this paper we provide a new proof of Theorem 1.2. It is based on the ABP method applied to a linear Neumann problem. It was Robert McCann (in a personal communication around 2000) who pointed out that the first author's proof of the classical isoperimetric inequality also worked in the anisotropic case. 


\subsection{Results}

The main result of the present paper, Theorem 1.3 below, is a weighted isoperimetric inequality which extends the two previous classical inequalities (Theorems 1.1 and 1.2). In particular, in Section 4 we will give a new proof of the classical Wulff theorem (for smooth domains) using the ABP method.

Before stating our main result, let us define the weighted anisotropic perimeter relative to an open cone $\Sigma$. The weights $w$ that we consider will always be continuous functions in $\bar{\Sigma}$, positive and locally Lipschitz in $\Sigma$, and homogeneous of degree $\alpha \geq 0$. Given a gauge $H$ in $\mathbb{R}^{n}$ and a weight $w$, we define (following [2]) the weighted anisotropic perimeter relative to the cone $\Sigma$ by

$$
P_{w, H}(E ; \Sigma):=\sup \left\{\int_{E \cap \Sigma} \operatorname{div}(\sigma w) d x: \sigma \in X_{w, \Sigma}, \sigma(x) \in W \text { for } x \in \Sigma\right\},
$$

where $E \subset \mathbb{R}^{n}$ is any measurable set with finite Lebesgue measure and

$$
X_{w, \Sigma}:=\left\{\sigma \in\left(L^{\infty}(\Sigma)\right)^{n}: \operatorname{div}(\sigma w) \in L^{\infty}(\Sigma) \text { and } \sigma w=0 \text { on } \partial \Sigma\right\} .
$$

It is not difficult to see that

$$
P_{w, H}(E ; \Sigma)=\int_{\partial E \cap \Sigma} H(v(x)) w(x) d S
$$

whenever $E$ is smooth enough.

The definition of $P_{w, H}$ is the same as the one given in [2]. In their notation, we are taking $d \mu=w \chi_{\Sigma} d x$ and $X_{w, \Sigma}=X_{\mu}$.

Moreover, when $H$ is the Euclidean norm we denote

$$
P_{w}(E ; \Sigma):=P_{w,\|\cdot\|_{2}}(E ; \Sigma) .
$$

When $w \equiv 1$ in $\Sigma$ and $H$ is the Euclidean norm we recover the definition of $P(E ; \Sigma)$ (see [2]).

Given a measurable set $F \subset \Sigma$, we denote by $w(F)$ the weighted volume of $F$,

$$
w(F):=\int_{F} w d x
$$

We also denote

$$
D=n+\alpha
$$

Note that the Wulff shape $W$ is independent of the weight $w$. Next we use the fact that if $v$ is the unit outward normal to $W \cap \Sigma$, then $x \cdot v(x)=H(v(x))$ a.e. on $\partial W \cap \Sigma$, $x \cdot v(x)=0$ a.e. on $\bar{W} \cap \partial \Sigma$, and $x \cdot \nabla w(x)=\alpha w(x)$ in $\Sigma$. This last equality follows from the degree $\alpha$ homogeneity of $w$. Then, with a similar argument to (1.11), we find

$$
\begin{aligned}
P_{w, H}(W ; \Sigma) & =\int_{\partial W \cap \Sigma} H(v(x)) w(x) d S=\int_{\partial W \cap \Sigma} x \cdot v(x) w(x) d S \\
& =\int_{\partial(W \cap \Sigma)} x \cdot v(x) w(x) d S=\int_{W \cap \Sigma} \operatorname{div}(x w(x)) d x \\
& =\int_{W \cap \Sigma}\{n w(x)+x \cdot \nabla w(x)\} d x=D w(W \cap \Sigma) .
\end{aligned}
$$


Here-and in our main result that follows - for all quantities to make sense we need to assume that $W \cap \Sigma \neq \emptyset$. Recall that both $W$ and $\Sigma$ are open convex sets, but $W \cap \Sigma=\varnothing$ may happen. This occurs for instance if $\left.H\right|_{\mathbb{S}^{n-1} \cap \Sigma} \equiv 0$. On the other hand, if $H>0$ on all $\mathbb{S}^{n-1}$ then $W \cap \Sigma \neq \varnothing$.

The following is our main result.

Theorem 1.3. Let $H$ be a gauge in $\mathbb{R}^{n}$, i.e., a function satisfying (1.6), and $W$ its associated Wulff shape defined by (1.8). Let $\Sigma$ be an open convex cone in $\mathbb{R}^{n}$ with vertex at the origin and such that $W \cap \Sigma \neq \emptyset$. Let $w$ be a continuous function in $\bar{\Sigma}$, positive in $\Sigma$, and positively homogeneous of degree $\alpha \geq 0$. Assume in addition that $w^{1 / \alpha}$ is concave in $\Sigma$ in case $\alpha>0$. Then, for each measurable set $E \subset \mathbb{R}^{n}$ with $w(E \cap \Sigma)<\infty$,

$$
\frac{P_{w, H}(E ; \Sigma)}{w(E \cap \Sigma)^{(D-1) / D}} \geq \frac{P_{w, H}(W ; \Sigma)}{w(W \cap \Sigma)^{(D-1) / D}},
$$

where $D=n+\alpha$.

Remark 1.4. Our key hypothesis that $w^{1 / \alpha}$ is a concave function is equivalent to a natural curvature-dimension bound (in fact, to the nonnegativity of the Bakry-Émery Ricci tensor in dimension $D=n+\alpha$ ). This was suggested to us by Cédric Villani, and has also been noticed by Cañete and Rosales [12, Lemma 3.9]. More precisely, we see the cone $\Sigma \subset \mathbb{R}^{n}$ as a Riemannian manifold of dimension $n$ equipped with a reference measure $w(x) d x$. We are also given a "dimension" $D=n+\alpha$. Consider the Bakry-Émery Ricci tensor, defined by

$$
\operatorname{Ric}_{D, w}=\operatorname{Ric}-\nabla^{2} \log w-\frac{1}{D-n} \nabla \log w \otimes \nabla \log w .
$$

Now, our assumption of $w^{1 / \alpha}$ being concave is equivalent to

$$
\operatorname{Ric}_{D, w} \geq 0 \text {. }
$$

Indeed, since Ric $\equiv 0$ and $D-n=\alpha,(1.16)$ reads

$$
-\nabla^{2} \log w^{1 / \alpha}-\nabla \log w^{1 / \alpha} \otimes \nabla \log w^{1 / \alpha} \geq 0,
$$

which is the same condition as $w^{1 / \alpha}$ being concave. Condition (1.16) is called a curvaturedimension bound; in the terminology of [52] we say that $\operatorname{CD}(0, D)$ is satisfied by $\Sigma \subset \mathbb{R}^{n}$ with the reference measure $w(x) d x$.

Note that the shape of the minimizer is $W \cap \Sigma$, and that $W$ depends only on $H$ and not on the weight $w$ or the cone $\Sigma$. In particular, in the isotropic case $H=\|\cdot\|_{2}$ we find the following noteworthy fact. Even though the weights we consider are not radial (unless $w \equiv 1$ ), Euclidean balls centered at the origin (intersected with the cone) still minimize this isoperimetric quotient. Recall that Euclidean balls centered at 0 have constant generalized mean curvature when the weight is homogeneous, as pointed out in (1.2), and thus they are candidates to minimize perimeter for a given volume. Our proof establishes that this is indeed true if in addition $w^{1 / \alpha}$ is concave.

Note also that we allow $w$ to vanish somewhere (or everywhere) on $\partial \Sigma$. 
Equality in (1.15) holds whenever $E \cap \Sigma=r W \cap \Sigma$, where $r$ is any positive number. However, in this paper we do not prove that $W \cap \Sigma$ is the unique minimizer of (1.15). The reason is that our proof involves the solution of an elliptic equation and, due to an important regularity issue, we need to approximate the given set $E$ by smooth sets. In a future work with E. Cinti and A. Pratelli we will refine the analysis in the proof of the present article and obtain a quantitative version of our isoperimetric inequality in cones. In particular, we will deduce uniqueness of minimizers (up to sets of measure zero). The quantitative version will be proved by using the techniques of the present paper (the ABP method applied to a linear Neumann problem) together with the ideas of Figalli-MaggiPratelli [24].

In the isotropic case, a recent result of Cañete and Rosales [12] deals with the same class of weights as ours. They allow not only positive homogeneities $\alpha>0$, but also negative ones $\alpha \leq-(n-1)$. They prove that if a smooth, compact, and orientable hypersurface in a smooth convex cone is stable for weighted perimeter (under variations preserving weighted volume), then it must be a sphere centered at the vertex of the cone. In [12] the stability of such spheres is proved for $\alpha \leq-(n-1)$, but not for $\alpha>0$. However, as pointed out in [12], when $\alpha>0$ their result combined with ours shows that spheres centered at the vertex are the unique minimizers among smooth hypersurfaces.

Theorem 1.3 contains the classical isoperimetric inequality, its version for convex cones, and the classical Wulff inequality. Indeed, taking $w \equiv 1, \Sigma=\mathbb{R}^{n}$, and $H=$ $\|\cdot\|_{2}$, we recover the classical isoperimetric inequality with optimal constant. Still taking $w \equiv 1$ and $H=\|\cdot\|_{2}$, but now letting $\Sigma$ be any open convex cone of $\mathbb{R}^{n}$, we have the isoperimetric inequality in convex cones of Lions and Pacella (Theorem 1.1). Moreover, if we take $w \equiv 1$ and $\Sigma=\mathbb{R}^{n}$ but let $H$ be some other gauge, we obtain the Wulff inequality (Theorem 1.2).

Remark 1.5. Let $w$ be a homogeneous weight of degree $\alpha$, and consider the isotropic isoperimetric problem in a cone $\Sigma \subset \mathbb{R}^{n}$. Then, by the proofs of [46, Proposition 3.6 and Lemma 3.8] the set $B_{1}(0) \cap \Sigma$ is stable if and only if

$$
\int_{\mathbb{S}^{n-1} \cap \Sigma}\left|\nabla_{\mathbb{S}^{n-1}} u\right|^{2} w d S \geq(n-1+\alpha) \int_{\mathbb{S}^{n-1} \cap \Sigma}|u|^{2} w d S
$$

for all functions $u \in C_{c}^{\infty}\left(\mathbb{S}^{n-1} \cap \Sigma\right)$ satisfying

$$
\int_{\mathbb{S}^{n-1} \cap \Sigma} u w d S=0 .
$$

Stability being a necessary condition for minimality, from Theorem 1.3 we deduce that if $\alpha>0$ and $\Sigma$ is convex, and $w^{1 / \alpha}$ is concave in $\Sigma$, then (1.17) holds.

In Theorem 1.3 we assume that $w$ is homogeneous of degree $\alpha$. In our proof, this assumption is essential in order that the paraboloid in (3.4) solves the PDE in (3.2), as explained in Section 3. Due to the homogeneity of $w$, the exponent $D=n+\alpha$ can be found just by a scaling argument in our inequality (1.15). Note that this exponent $D$ has a dimension flavor if one compares (1.15) with (1.5) or (1.12). Also, it is the volume growth exponent in the sense that $w\left(B_{r}(0) \cap \Sigma\right)=C r^{D}$ for all $r>0$. The interpretation of $D$ as 
a dimension is more clear in the case $w(x)=x_{1}^{A_{1}} \cdots x_{n}^{A_{n}}, A_{i}>0$, and $\Sigma=(0, \infty)^{n}$. The Sobolev and isoperimetric inequalities with such monomial weights were studied by the first two authors $[8,9]$. When all the exponents $A_{i}$ are positive integers, the weighted isoperimetric inequality in $\mathbb{R}^{n}$ is equivalent to the (unweighted) isoperimetric inequality in $\mathbb{R}^{D}$ with $D=n+A_{1}+\cdots+A_{n}$ (see [9] for more details).

An immediate consequence of Theorem 1.3 is the following weighted isoperimetric inequality in $\mathbb{R}^{n}$ for symmetric sets and even weights. It follows from our main result by taking $\Sigma=(0, \infty)^{n}$.

Corollary 1.6. Let $w$ be a nonnegative continuous function in $\mathbb{R}^{n}$, even with respect to each variable, homogeneous of degree $\alpha>0$, and such that $w^{1 / \alpha}$ is concave in $(0, \infty)^{n}$. Let $E \subset \mathbb{R}^{n}$ be any measurable set, symmetric with respect to each coordinate hyperplane $\left\{x_{i}=0\right\}$, and with $|E|<\infty$. Then

$$
\frac{P_{w}\left(E ; \mathbb{R}^{n}\right)}{|E|^{(D-1) / D}} \geq \frac{P_{w}\left(B_{1} ; \mathbb{R}^{n}\right)}{\left|B_{1}\right|^{(D-1) / D}},
$$

where $D=n+\alpha$ and $B_{1}$ is the unit ball in $\mathbb{R}^{n}$.

The symmetry assumption in Corollary 1.6 is satisfied in some applications arising in nonlinear problems, such as the one in [8]. Without this assumption, isoperimetric sets in (1.19) may not be balls. For example, for the monomial weight $w(x)=\left|x_{1}\right|^{A_{1}} \cdots\left|x_{n}\right|^{A_{n}}$ in $\mathbb{R}^{n}$ with all $A_{i}$ positive, $B_{1} \cap(0, \infty)^{n}$ is an isoperimetric set, while the whole ball $B_{r}$ having the same weighted volume as $B_{1} \cap(0, \infty)^{n}$ is not an isoperimetric set (since it has larger perimeter).

We know only of few results where nonradial weights lead to radial minimizers. The first one is the isoperimetric inequality by Maderna-Salsa [34] in the upper half-plane $\mathbb{R}_{+}^{2}$ with the weight $x_{2}^{\alpha}, \alpha>0$. To establish their isoperimetric inequality, they first proved the existence of a minimizer for the perimeter functional under constraint of fixed area, then computed the first variation of this functional, and finally solved the resulting ODE to find all minimizers. The second result is due to Brock-Chiacchio-Mercaldo [4] and extends the one in [34] by including the weights $x_{n}^{\alpha} \exp \left(c|x|^{2}\right)$ in $\mathbb{R}_{+}^{n}$, with $\alpha \geq 0$ and $c \geq 0$. In both papers it is proved that half-balls centered at the origin are minimizers of the isoperimetric quotient with these weights. Another one, of course, is our isoperimetric inequality with monomial weights [9] explained above. At the same time as ourselves, but using totally different methods, Brock, Chiacchio, and Mercaldo [5] proved an isoperimetric inequality in $\Sigma=\left\{x_{1}>0, \ldots, x_{n}>0\right\}$ with the weight $x_{1}^{A_{1}} \cdots x_{n}^{A_{n}} \exp \left(c|x|^{2}\right)$ with $A_{i} \geq 0$ and $c \geq 0$.

In all these results, although the weight $x_{1}^{A_{1}} \cdots x_{n}^{A_{n}}$ is not radial, it has a very special structure. Indeed, when all $A_{1}, \ldots, A_{n}$ are nonnegative integers, the isoperimetric problem with the weight $x_{1}^{A_{1}} \cdots x_{n}^{A_{n}}$ is equivalent to the isoperimetric problem in $\mathbb{R}^{n+A_{1}+\cdots+A_{n}}$ for sets that have symmetry of revolution with respect to the first $A_{1}+1$ variables, the next $A_{2}+1$ variables, ..., and so on until the last $A_{n}+1$ variables. By this observation, the fact that half-balls centered at the origin are minimizers in $\mathbb{R}_{+}^{n}$ with the weight $x_{1}^{A_{1}} \cdots x_{n}^{A_{n}}$ or $x_{1}^{A_{1}} \cdots x_{n}^{A_{n}} \exp \left(c|x|^{2}\right)$, for $c \geq 0$ and $A_{i}$ nonnegative integers, 
follows from the isoperimetric inequality in $\mathbb{R}^{n+A_{1}+\cdots+A_{n}}$ with the weight $\exp \left(c|x|^{2}\right)$, $c \geq 0$ (which is a radial weight). Thus, it was reasonable to expect that the same result for noninteger exponents $A_{1}, \ldots, A_{n}$ would also hold—as it does.

After announcing our result and proof in [10], Emanuel Milman showed us a nice geometric construction that yields the particular case when $\alpha$ is a nonnegative integer in our weighted inequality of Theorem 1.3. Using this construction, the weighted inequality in a convex cone is obtained as a limit case of the unweighted Lions-Pacella inequality in a narrow cone of $\mathbb{R}^{n+\alpha}$. We reproduce it in Remark 5.3-see also the blog of Frank Morgan [42].

More recently, after posting the preprint version of the present paper, Milman and Rotem [39] have found an alternative proof of our isoperimetric inequalitiy, Theorem 1.3 above ([39] mentions that the same has been found independenly by Nguyen). Their proof uses the Borell-Brascamp-Lieb extension of the the Brunn-Minkowski inequality.

\subsection{Sobolev inequalities with best constant}

Our result leads to new weighted Sobolev inequalities in convex cones of $\mathbb{R}^{n}$. In addition, we can compute their optimal constants, as well as the shape of the extremizers. Indeed, given any smooth function $u$ with compact support in $\mathbb{R}^{n}$ (we do not assume that $u$ vanishes on $\partial \Sigma$ ), one uses the coarea formula and Theorem 1.3 applied to each level set of $u$. This establishes the Sobolev inequality (1.3) for $p=1$. The constant $C_{w, 1, n}$ obtained in this way is optimal, and coincides with the best constant in our isoperimetric inequality (1.19).

When $1<p<D$, Theorem 1.3 also leads to the Sobolev inequality (1.3) with best constant. This is a consequence of our isoperimetric inequality and a weighted radial rearrangement of Talenti [47], since these two results yield the radial symmetry of minimizers. See [9] for details in the case of monomial weights $w(x)=\left|x_{1}\right|^{A_{1}} \cdots\left|x_{n}\right|^{A_{n}}$.

If we use Corollary 1.6 instead of Theorem 1.3, with the same argument we find the Sobolev inequality

$$
\left(\int_{\mathbb{R}^{n}}|u|^{p_{*}} w(x) d x\right)^{1 / p_{*}} \leq C_{w, p, n}\left(\int_{\mathbb{R}^{n}}|\nabla u|^{p} w(x) d x\right)^{1 / p},
$$

where $p_{*}=p D /(D-p), D=n+\alpha$, and $1 \leq p<D$. Here, $w$ is any weight satisfying the hypotheses of Corollary 1.6, and $u$ is any smooth function with compact support in $\mathbb{R}^{n}$ which is symmetric with respect to each variable $x_{i}, i=1, \ldots, n$.

\subsection{The proof. Related works}

The proof of Theorem 1.3 consists in applying the ABP method to a linear Neumann problem involving the operator $w^{-1} \operatorname{div}(w \nabla u)$, where $w$ is the weight. When $w \equiv 1$, the idea goes back to 2000 in the works $[6,7]$ of the first author, where the classical isoperimetric inequality in all of $\mathbb{R}^{n}$ (here $w \equiv 1$ ) was proved with a new method. It 
consisted in solving the problem

$$
\begin{cases}\Delta u=b_{\Omega} & \text { in } \Omega, \\ \frac{\partial u}{\partial v}=1 & \text { on } \partial \Omega,\end{cases}
$$

for a certain constant $b_{\Omega}$, to produce a bijective map with the gradient of $u, \nabla u$ : $\Gamma_{u, 1} \rightarrow B_{1}$, which leads to the isoperimetric inequality. Here $\Gamma_{u, 1} \subset \Gamma_{u} \subset \Omega$ and $\Gamma_{u, 1}$ is a certain subset of the lower contact set $\Gamma_{u}$ of $u$ (see Section 3 for details). The use of the ABP method is crucial in the proof.

Previously, Trudinger [51] had given a proof of the classical isoperimetric inequality in 1994, using the theory of Monge-Ampère equations and the ABP estimate. His proof consists in applying the ABP estimate to the Monge-Ampère problem

$$
\begin{cases}\operatorname{det} D^{2} u=\chi_{\Omega} & \text { in } B_{R}, \\ u=0 & \text { on } \partial B_{R},\end{cases}
$$

where $\chi_{\Omega}$ is the characteristic function of $\Omega$ and $B_{R}=B_{R}(0)$, and then letting $R \rightarrow \infty$.

Before these two works ([51] and [6]), there was already a proof of the isoperimetric inequality using a certain map (or coupling). This is Gromov's proof, which used the Knothe map (see [52]).

After these three proofs, in 2004 Cordero-Erausquin, Nazaret, and Villani [18] used the Brenier map from optimal transportation to give a beautiful proof of the anisotropic isoperimetric inequality (see also [52]). More recently, Figalli-Maggi-Pratelli [24] established a sharp quantitative version of the anisotropic isoperimetric inequality, also using the Brenier map. In the case of the Lions-Pacella isoperimetric inequality, this has been done by Figalli-Indrei [22] very recently. As mentioned before, the proof in the present article is also suited for a quantitative version, as we will show in a future work with Cinti and Pratelli.

After finding the proof of our result given in this paper (which uses the ABP technique), we realized that optimal transportation techniques could also be used to prove the result. In the case of the half-space $\left\{x_{n}>0\right\}$ equipped with the weight $x_{n}^{\alpha}$, the same was realized by A. Figalli.

Regarding isoperimetric inequalities on manifolds, in the Bibliographical Notes to Chapter 21 of C. Villani's book [52], he points out that optimal transport techniques might be used to prove isoperimetric inequalities on cones with nonnegative Ricci curvature.

\subsection{Plan of the paper}

The rest of the article is organized as follows. In Section 2 we give examples of weights to which our result applies. In Section 3 we introduce the elements appearing in the proof of Theorem 1.3. To illustrate these ideas, in Section 4 we give the proof of the classical Wulff theorem via the ABP method. Finally, in Section 5 we prove Theorem 1.3. 


\section{Examples of weights}

When $w \equiv 1$, our main result yields the classical isoperimetric inequality, its version for convex cones, and also the Wulff theorem. On the other hand, given an open convex cone $\Sigma \subset \mathbb{R}^{n}$ (different than the whole space and a half-space), there is a large family of functions that are homogeneous of degree one and concave in $\Sigma$. Any positive power of one of these functions is an admissible weight for Theorem 1.3. Next we give some concrete examples of weights $w$ to which our result applies.

(i) Assume that $w_{1}$ and $w_{2}$ are concave homogeneous functions of degree one in an open convex cone $\Sigma$. Then $w_{1}^{a} w_{2}^{b}$ with $a \geq 0$ and $b \geq 0$, and $\left(w_{1}^{r}+w_{2}^{r}\right)^{\alpha / r}$ with $r \in(0,1]$ or $r<0$, satisfy the hypotheses of Theorem 1.3.

(ii) For any open convex cone $\Sigma$ and any $\alpha \geq 0$,

$$
w(x)=\operatorname{dist}(x, \partial \Sigma)^{\alpha}
$$

is an admissible weight. When the cone is $\Sigma=\left\{x_{i}>0, i=1, \ldots, n\right\}$, this weight is exactly $\min \left\{x_{1}, \ldots, x_{n}\right\}^{\alpha}$.

(iii) If the concavity condition is satisfied by a weight $w$ in a convex cone $\Sigma^{\prime}$ then it is also satisfied in any convex subcone $\Sigma \subset \Sigma^{\prime}$. Note that this gives examples of weights $w$ and cones $\Sigma$ in which $w$ is positive on $\partial \Sigma \backslash\{0\}$.

(iv) Let $\Sigma_{1}, \ldots, \Sigma_{k}$ be convex cones and $\Sigma=\Sigma_{1} \cap \cdots \cap \Sigma_{k}$. Let

$$
\delta_{i}(x)=\operatorname{dist}\left(x, \partial \Sigma_{i}\right) .
$$

Then the weight

$$
w(x)=\delta_{1}^{A_{1}} \cdots \delta_{k}^{A_{k}}, \quad x \in \Sigma,
$$

with $A_{1} \geq 0, \ldots, A_{k} \geq 0$ satisfies the hypotheses of Theorem 1.3. This follows from (i)-(iii). When $k=n$ and $\Sigma_{i}=\left\{x_{i}>0\right\}, i=1, \ldots, n$, then $\Sigma=\left\{x_{1}>0\right.$, $\left.\ldots, x_{n}>0\right\}$ and we obtain the monomial weight $w(x)=x_{1}^{A_{1}} \cdots x_{n}^{A_{n}}$.

(v) In the cone $\Sigma=(0, \infty)^{n}$, the weights

$$
w(x)=\left(A_{1} x_{1}^{1 / p}+\cdots+A_{n} x_{n}^{1 / p}\right)^{\alpha p}
$$

for $p \geq 1, A_{i} \geq 0$, and $\alpha>0$ satisfy the hypotheses of Theorem 1.3. Similarly, one may take the same weight but with $p<0$. This can be shown by using the Minkowski inequality.

(vi) Powers of hyperbolic polynomials also provide examples of weights (see for example [28] or [11, Section 1]). For any hyperbolic polynomial $P$, the weight

$$
w(x)=P(x)^{\alpha / k}
$$

satisfies the hypotheses of Theorem 1.3. Typical examples of hyperbolic polynomials are

$$
P(x)=x_{1}^{2}-\lambda_{2} x_{2}^{2}-\cdots-\lambda_{n} x_{n}^{2} \quad \text { in } \Sigma=\left\{x_{1}>\sqrt{\lambda_{2} x_{2}^{2}+\cdots+\lambda_{n} x_{n}^{2}}\right\},
$$

with $\lambda_{2}>0, \ldots, \lambda_{n}>0$, or the elementary symmetric functions

$$
\sigma_{k}(x)=\sum_{1 \leq i_{1}<\cdots<i_{k} \leq n} x_{i_{1}} \cdots x_{i_{k}} \quad \text { in } \Sigma=\left\{\sigma_{1}>0, \ldots, \sigma_{k}>0\right\} .
$$


(vii) If $\sigma_{k}$ and $\sigma_{l}$ are the elementary symmetric functions of degree $k$ and $l$ with $1 \leq$ $k<l \leq n$, then $\left(\sigma_{l} / \sigma_{k}\right)^{1 /(l-k)}$ is concave in the cone $\Sigma=\left\{\sigma_{1}>0, \ldots, \sigma_{k}>0\right\}$ (see [35]). In particular, setting $k=n$ and $l=1$ we find that we can take

$$
w(x)=\left(\frac{x_{1} \cdots x_{n}}{x_{1}+\cdots+x_{n}}\right)^{\alpha /(n-1)}
$$

in Theorem 1.3 or in Corollary 1.6.

(viii) If $f: \mathbb{R} \rightarrow \mathbb{R}_{+}$is any continuous function which is concave in $(a, b)$, then $w(x)=$ $x_{1} f\left(x_{2} / x_{1}\right)$ is an admissible weight in $\Sigma=\{x=(r, \theta): \arctan a<\theta<\arctan b\}$. In particular, in the cone $\Sigma=(0, \infty)^{2} \subset \mathbb{R}^{2}$ one may take

$$
w(x)=\left(\frac{x_{1}-x_{2}}{\log x_{1}-\log x_{2}}\right)^{\alpha} \quad \text { or } \quad w(x)=\frac{x^{a+1} y^{b+1}}{\left(x^{p}+y^{p}\right)^{1 / p}}
$$

with $\alpha>0, a, b \geq 0$, and $p>-1$.

\section{Description of the proof}

The proof of Theorem 1.3 follows the ideas introduced by the first author in a new proof of the classical isoperimetric inequality; see $[6,7]$ or the last edition of Chavel's book [15]. This proof uses the ABP method, as explained next.

The Alexandroff-Bakelman-Pucci (or ABP) estimate is an $L^{\infty}$ bound for solutions of the Dirichlet problem associated to second order uniformly elliptic operators written in nondivergence form,

$$
L u=a_{i j}(x) \partial_{i j} u+b_{i}(x) \partial_{i} u+c(x) u,
$$

with bounded measurable coefficients in a domain $\Omega$ of $\mathbb{R}^{n}$. It asserts that if $\Omega$ is bounded and $c \leq 0$ in $\Omega$ then, for every $u \in C^{2}(\Omega) \cap C(\bar{\Omega})$,

$$
\sup _{\Omega} u \leq \sup _{\partial \Omega} u+C \operatorname{diam}(\Omega)\|L u\|_{L^{n}(\Omega)},
$$

where $C$ is a constant depending only on the ellipticity constants of $L$ and on the $L^{n}$ norm of the coefficients $b_{i}$. The estimate was proven by the above mentioned authors in the sixties using a technique that is nowadays called "the ABP method". See [7] and references therein for more information on this estimate.

The proof of the classical isoperimetric inequality in [6, 7] consists in applying the ABP method to an appropriate Neumann problem for the Laplacian-instead of applying it to a Dirichlet problem as is customary. Namely, to estimate $P(\Omega) /|\Omega|^{(n-1) / n}$ from below for a smooth domain $\Omega$ (where $P(\Omega)$ is the perimeter of $\Omega$ ), one considers the problem

$$
\begin{cases}\Delta u=b_{\Omega} & \text { in } \Omega, \\ \frac{\partial u}{\partial v}=1 & \text { on } \partial \Omega .\end{cases}
$$

The constant $b_{\Omega}=P(\Omega) /|\Omega|$ is chosen so that the problem has a solution. Next, one proves that $B_{1} \subset \nabla u\left(\Gamma_{u}\right)$ with a contact argument (for a certain "contact" set $\Gamma_{u} \subset \Omega$ ), 
and then one estimates the measure of $\nabla u\left(\Gamma_{u}\right)$ by using the area formula and the inequality between the geometric and arithmetic means. Note that the solution of (3.1) is

$$
u(x)=|x|^{2} / 2 \quad \text { when } \Omega=B_{1},
$$

and in this case one verifies that all the inequalities appearing in this ABP argument are equalities. After having proved the isoperimetric inequality for smooth domains, a standard approximation argument extends it to all sets of finite perimeter.

As pointed out by R. McCann, the same method also yields the Wulff theorem. For this, one replaces the Neumann data in (3.1) by $\partial u / \partial v=H(v)$ and uses the same argument explained above. This proof of the Wulff theorem is given in Section 4.

We now sketch the proof of Theorem 1.3 in the isotropic case, that is, when $H=\|\cdot\|_{2}$. In this case, optimizers are Euclidean balls centered at the origin intersected with the cone $\Sigma$. First, we assume that $E=\Omega$ is a bounded smooth domain inside the convex cone $\Sigma$. The key idea is to consider a similar problem to (3.1) but where the Laplacian is replaced by the operator

$$
w^{-1} \operatorname{div}(w \nabla u)=\Delta u+\frac{\nabla w}{w} \cdot \nabla u .
$$

Essentially (but, as we will see, this is not exactly as we proceed—because of a regularity issue), we solve the following Neumann problem in $\Omega \subset \Sigma$ :

$$
\begin{cases}w^{-1} \operatorname{div}(w \nabla u)=b_{\Omega} & \text { in } \Omega, \\ \frac{\partial u}{\partial v}=1 & \text { on } \partial \Omega \cap \Sigma, \\ \frac{\partial u}{\partial v}=0 & \text { on } \partial \Omega \cap \partial \Sigma,\end{cases}
$$

where the constant $b_{\Omega}$ is again chosen depending on weighted perimeter and volume so that the problem admits a solution. Whenever $u$ belongs to $C^{1}(\bar{\Omega})$ - which is not always the case, as discussed below in this section — by touching the graph of $u$ from below with planes (as in the proof of the classical isoperimetric inequality explained above) we find that

$$
B_{1} \cap \Sigma \subset \nabla u(\Omega) .
$$

Then, using the area formula, an appropriate weighted geometric-arithmetic means inequality, and the concavity condition on the weight $w$, we obtain our weighted isoperimetric inequality. Note that the solution of (3.2) is

$$
u(x)=|x|^{2} / 2 \quad \text { when } \Omega=B_{1} \cap \Sigma .
$$

In this case, all the chain of inequalities in our proof become equalities, and this yields the sharpness of the result.

In the previous argument there is an important technical difficulty that comes from the possible lack of regularity up to the boundary of the solution to the weighted Neumann problem (3.2). For instance, if $\Omega \cap \Sigma$ is a smooth domain that has some part of its boundary 
lying on $\partial \Sigma$-and hence $\partial \Omega$ meets $\partial \Sigma$ tangentially-then $u$ cannot be $C^{1}$ up to the boundary. This is because the Neumann condition itself is not continuous and hence $\partial_{\nu} u$ would jump from 1 to one 0 where $\partial \Omega$ meets $\partial \Sigma$.

The fact that $u$ could not be $C^{1}$ up to the boundary prevents us from using our contact argument to prove (3.3). Nevertheless, the argument sketched above does work for smooth domains $\Omega$ well contained in $\Sigma$, that is, satisfying $\bar{\Omega} \subset \Sigma$. If $w \equiv 0$ on $\partial \Sigma$ this is enough to deduce the inequality for all measurable sets $E$ by an approximation argument. However, if $w>0$ at some part of (or everywhere on) $\partial \Sigma$, it is not always possible to find sequences of smooth sets with closure contained in the open cone and approximating a given measurable set $E \subset \Sigma$ in relative perimeter. This is because the relative perimeter does not count the part of the boundary of $E$ which lies on $\partial \Sigma$. To get around this difficulty (recall that we are describing the proof in the isotropic case, $H \equiv 1$ ) we consider an anisotropic problem in $\mathbb{R}^{n}$ for which approximation is possible. Namely, we choose a gauge $H_{0}$ defined as the gauge associated to the convex set $B_{1} \cap \Sigma$ (see (1.9)). Then we prove that $P_{w, H_{0}}(\cdot ; \Sigma)$ is a calibration of the functional $P_{w}(\cdot ; \Sigma)$, in the following sense. For all $E \subset \Sigma$ we will have

$$
P_{w, H_{0}}(E ; \Sigma) \leq P_{w}(E ; \Sigma)
$$

while for $E=B_{1} \cap \Sigma$,

$$
P_{w, H_{0}}\left(B_{1} ; \Sigma\right)=P_{w}\left(B_{1} \cap \Sigma ; \Sigma\right) .
$$

As a consequence, the isoperimetric inequality with perimeter $P_{w, H_{0}}(\cdot ; \Sigma)$ implies the one with $P_{w}(\cdot ; \Sigma)$. For $P_{w, H_{0}}(\cdot ; \Sigma)$ approximation results are available and, as in the case of $w \equiv 0$ on $\partial \Sigma$, it is enough to consider smooth sets satisfying $\bar{\Omega} \subset \Sigma$-for which there are no regularity problems with the solution of the elliptic problem.

To prove Theorem 1.3 for a general anisotropic perimeter $P_{w, H}(\cdot ; \Sigma)$ we also consider a "calibrated" perimeter $P_{w, H_{0}}(\cdot ; \Sigma)$, where $H_{0}$ is now the gauge associated to the convex set $W \cap \Sigma$.

\section{Proof of the classical Wulff inequality}

In this section we prove the classical Wulff theorem for smooth domains by using the ideas introduced by the first author in $[6,7]$. When $H$ is smooth on $\mathbb{S}^{n-1}$, we also show that the Wulff shapes are the only smooth sets for which equality is attained.

Proof of Theorem 1.2. We prove the Wulff inequality only for smooth domains, which we denote by $\Omega$ instead of $E$. By approximation, if (1.12) holds for all smooth domains then it holds for all sets of finite perimeter.

By regularizing $H$ on $\mathbb{S}^{n-1}$ and then extending it homogeneously, we can assume that $H$ is smooth in $\mathbb{R}^{n} \backslash\{0\}$. For nonsmooth $H$ this approximation argument will yield inequality (1.12), but not the equality cases. 
Let $u$ be a solution of the Neumann problem

$$
\begin{cases}\Delta u=\frac{P_{H}(\Omega)}{|\Omega|} & \text { in } \Omega, \\ \frac{\partial u}{\partial v}=H(v) & \text { on } \partial \Omega,\end{cases}
$$

where $\Delta$ denotes the Laplace operator and $\partial u / \partial v$ the exterior normal derivative of $u$ on $\partial \Omega$. Recall that $P_{H}(\Omega)=\int_{\partial \Omega} H(v(x)) d S$. The constant $P_{H}(\Omega) /|\Omega|$ has been chosen so that the problem has a unique solution up to an additive constant. Since $\left.H\right|_{\mathbb{S}^{n-1}}$ and $\Omega$ are smooth, we deduce that $u$ is smooth in $\bar{\Omega}$. See [44] for a recent exposition of these classical facts and for a new Schauder estimate for (4.1).

Consider the lower contact set of $u$, defined by

$$
\Gamma_{u}=\{x \in \Omega: u(y) \geq u(x)+\nabla u(x) \cdot(y-x) \text { for all } y \in \bar{\Omega}\} .
$$

It is the set of points where the tangent hyperplane to the graph of $u$ lies below $u$ in all $\bar{\Omega}$. We claim that

$$
W \subset \nabla u\left(\Gamma_{u}\right),
$$

where $W$ denotes the Wulff shape associated to $H$, given by (1.8).

To show (4.3), take any $p \in W$, i.e., any $p \in \mathbb{R}^{n}$ satisfying

$$
p \cdot v<H(v) \quad \text { for all } v \in \mathbb{S}^{n-1} .
$$

Let $x \in \bar{\Omega}$ be a point such that

$$
\min _{y \in \bar{\Omega}}\{u(y)-p \cdot y\}=u(x)-p \cdot x
$$

(this is, up to a sign, the Legendre transform of $u$ ). If $x \in \partial \Omega$ then the exterior normal derivative of $u(y)-p \cdot y$ at $x$ would be nonpositive, and hence $(\partial u / \partial v)(x) \leq p \cdot v<H(v)$, contrary to the boundary condition of (4.1). It follows that $x \in \Omega$, and therefore $x$ is an interior minimum point of the function $u(y)-p \cdot y$. In particular, $p=\nabla u(x)$ and $x \in \Gamma_{u}$. Claim (4.3) is now proved. It is interesting to visualize geometrically the proof of the claim, by considering the graphs of the functions $p \cdot y+c$ for $c \in \mathbb{R}$. These are parallel hyperplanes which lie, for $c$ close to $-\infty$, below the graph of $u$. We let $c$ increase and consider the first $c$ for which there is contact or "touching" at a point $x$. It is clear geometrically that $x \notin \partial \Omega$, since $p \cdot v<H(v)$ for all $v \in \mathbb{S}^{n-1}$ and $\partial u / \partial v=H(v)$ on $\partial \Omega$.

Now, from (4.3) we deduce

$$
|W| \leq\left|\nabla u\left(\Gamma_{u}\right)\right|=\int_{\nabla u\left(\Gamma_{u}\right)} d p \leq \int_{\Gamma_{u}} \operatorname{det} D^{2} u(x) d x .
$$

We have applied the area formula to the smooth map $\nabla u: \Gamma_{u} \rightarrow \mathbb{R}^{n}$, and we have used the fact that its Jacobian, det $D^{2} u$, is nonnegative in $\Gamma_{u}$ by definition of this set. 
Next, we use the classical inequality between the geometric and the arithmetic means applied to the eigenvalues of $D^{2} u(x)$ (which are nonnegative numbers for $x \in \Gamma_{u}$ ). We obtain

$$
\operatorname{det} D^{2} u \leq\left(\frac{\Delta u}{n}\right)^{n} \quad \text { in } \Gamma_{u} .
$$

This, combined with (4.4) and $\Delta u \equiv P_{H}(\Omega) /|\Omega|$, gives

$$
|W| \leq\left(\frac{P_{H}(\Omega)}{n|\Omega|}\right)^{n}\left|\Gamma_{u}\right| \leq\left(\frac{P_{H}(\Omega)}{n|\Omega|}\right)^{n}|\Omega| .
$$

Finally, using $P_{H}(W)=n|W|$ —see (1.11)—we conclude that

$$
\frac{P_{H}(W)}{|W|^{(n-1) / n}}=n|W|^{1 / n} \leq \frac{P_{H}(\Omega)}{|\Omega|^{(n-1) / n}}
$$

Note that when $\Omega=W$, the solution of (4.1) is $u(x)=|x|^{2} / 2$ since $\Delta u=n$ and $u_{v}(x)=x \cdot v(x)=H(v(x))$ a.e. on $\partial W$-recall (1.10). In particular, $\nabla u=$ Id and all the eigenvalues of $D^{2} u(x)$ are equal. Therefore, it is clear that all inequalities (and inclusions) in (4.3)-(4.7) are equalities when $\Omega=W$. This explains why the proof gives the best constant in the inequality.

Let us see next that when $\left.H\right|_{\mathbb{S}^{n-1}}$ is smooth, the Wulff shaped domains $\Omega=a W+b$ are the only smooth domains for which equality occurs in (1.12). Indeed, if (4.7) is an equality then all the inequalities in (4.4)-(4.6) are also equalities. In particular, $\left|\Gamma_{u}\right|=|\Omega|$. Since $\Gamma_{u} \subset \Omega, \Omega$ is an open set, and $\Gamma_{u}$ is closed relative to $\Omega$, we deduce that $\Gamma_{u}=\Omega$.

Recall that the geometric and arithmetic means of $n$ nonnegative numbers are equal if and only if these $n$ numbers are all equal. Hence, the equality in (4.5) and the fact that $\Delta u$ is constant in $\Omega$ imply that $D^{2} u=a$ Id in all $\Gamma_{u}=\Omega$, where Id is the identity matrix and $a=P_{H}(\partial \Omega) /(n|\Omega|)$ is a positive constant. Let $x_{0} \in \Omega$ be any given point. Integrating $D^{2} u=a$ Id on segments from $x_{0}$, we deduce that

$$
u(x)=u\left(x_{0}\right)+\nabla u\left(x_{0}\right) \cdot\left(x-x_{0}\right)+\frac{a}{2}\left|x-x_{0}\right|^{2}
$$

for $x$ in a neighborhood of $x_{0}$. In particular, $\nabla u(x)=\nabla u\left(x_{0}\right)+a\left(x-x_{0}\right)$ in such a neighborhood, and hence the map $\nabla u-a \mathrm{I}$ is locally constant. Since $\Omega$ is connected, we deduce that the map $\nabla u-a \mathrm{I}$ is indeed a constant, say $\nabla u-a \mathrm{I} \equiv y_{0}$.

It follows that $\nabla u\left(\Gamma_{u}\right)=\nabla u(\Omega)=y_{0}+a \Omega$. By (4.3) we know that $W \subset \nabla u\left(\Gamma_{u}\right)=$ $y_{0}+a \Omega$. In addition, these two sets have the same measure since equalities occur in (4.4). Thus, $y_{0}+a \Omega$ is equal to $W$ up to a set of measure zero. In fact, in the present situation, since $W$ is convex and $y_{0}+a \Omega$ is open, one easily proves that $W=y_{0}+a \Omega$. Hence, $\Omega$ is of the form $\tilde{a} W+\tilde{b}$ for some $\tilde{a}>0$ and $\tilde{b} \in \mathbb{R}^{n}$. 


\section{Proof of Theorem 1.3}

In this section we prove Theorem 1.3. At the end of the section, we include the geometric argument of E. Milman that provides an alternative proof of Theorem 1.3 in the case where the exponent $\alpha$ is an integer.

In the proof we will use the following lemma.

Lemma 5.1. Let $w$ be a positive homogeneous function of degree $\alpha>0$ in an open cone $\Sigma \subset \mathbb{R}^{n}$. Then the following conditions are equivalent:

- For each $x, z \in \Sigma$,

$$
\alpha\left(\frac{w(z)}{w(x)}\right)^{1 / \alpha} \leq \frac{\nabla w(x) \cdot z}{w(x)} .
$$

- The function $w^{1 / \alpha}$ is concave in $\Sigma$.

Proof. Assume first $\alpha=1$. A function $w$ is concave in $\Sigma$ if and only if, for all $x, z \in \Sigma$,

$$
w(x)+\nabla w(x) \cdot(z-x) \geq w(z) .
$$

Now, since $w$ is homogeneous of degree 1 , we have

$$
\nabla w(x) \cdot x=w(x)
$$

This can be seen by differentiating the equality $w(t x)=t w(x)$ and evaluating at $t=1$. Hence, from (5.1) and (5.2) we deduce that a homogeneous function $w$ of degree 1 is concave if and only if

$$
w(z) \leq \nabla w(x) \cdot z
$$

This proves the lemma for $\alpha=1$.

Assume now $\alpha \neq 1$. Define $v=w^{1 / \alpha}$, and apply the result proved above to the function $v$, which is homogeneous of degree 1 . We find that $v$ is concave if and only if

$$
v(z) \leq \nabla v(x) \cdot z
$$

Therefore, since $\nabla v(x)=\alpha^{-1} w(x)^{1 / \alpha-1} \nabla w(x)$, we deduce that $w^{1 / \alpha}$ is concave if and only if

$$
w(z)^{1 / \alpha} \leq \frac{\nabla w(x) \cdot z}{\alpha w(x)^{1-1 / \alpha}},
$$

and the lemma follows.

We can now establish Theorem 1.3. As explained in Remark 5.2 below, the proof is substantially shorter when $w$ vanishes on $\partial \Sigma$. This fact will be pointed out along the proof, where we will refer to Remark 5.2.

Proof of Theorem 1.3. Let

$$
W_{0}:=W \cap \Sigma,
$$

an open convex set, and nonempty by assumption. Since $\lambda W_{0} \subset W_{0}$ for all $\lambda \in(0,1)$, we deduce that $0 \in \bar{W}_{0}$. Therefore, as discussed in Subsection 1.1, there is a unique 
gauge $H_{0}$ whose Wulff shape is $W_{0}$. In fact, $H_{0}$ is defined by expression (1.9) (with $W$ and $H$ replaced by $W_{0}$ and $H_{0}$ ).

Since $H_{0} \leq H$ we have

$$
P_{w, H_{0}}(E ; \Sigma) \leq P_{w, H}(E ; \Sigma) \quad \text { for each measurable set } E,
$$

while, in view of (1.13),

$$
P_{w, H_{0}}\left(W_{0} ; \Sigma\right)=P_{w, H}(W ; \Sigma) \quad \text { and } \quad w\left(W_{0}\right)=w(W \cap \Sigma) .
$$

Thus, it suffices to prove that

$$
\frac{P_{w, H_{0}}(E ; \Sigma)}{w(E)^{(D-1) / D}} \geq \frac{P_{w, H_{0}}\left(W_{0} ; \Sigma\right)}{w\left(W_{0}\right)^{(D-1) / D}}
$$

for all measurable sets $E \subset \Sigma$ with $w(E)<\infty$.

The definition of $H_{0}$ is motivated as follows. Note that $H_{0}$ vanishes on the directions normal to the cone $\Sigma$. Thus, by considering $H_{0}$ instead of $H$, we will be able (by an approximation argument) to assume that $E$ is a smooth domain whose closure is contained in $\Sigma$. This approximation cannot be done when $H$ does not vanish on the directions normal to the cone-since the relative perimeter does not count the part of the boundary lying on $\partial \Sigma$, while when $\bar{E} \subset \Sigma$, the whole perimeter is counted. When $H=\|\cdot\|_{2}$ and $w$ vanishes on $\partial \Sigma$ the weighted perimeter of a piece of surface that approaches $\partial \Sigma$ from the inside converges to zero (since $w(x) \rightarrow 0$ as $x \rightarrow \partial \Sigma$ ). This makes the proof simpler in this particular case, and there is no need to consider anisotropic gauges (see Remark 5.2).

We split the proof of (5.3) into three cases.

Case 1: $E=\Omega$, where $\Omega$ is a smooth domain satisfying $\bar{\Omega} \subset \Sigma$. At this stage, it is clear that by regularizing $\left.w\right|_{\bar{\Omega}}$ and $\left.H_{0}\right|_{\mathbb{S}^{n-1}}$ we can assume $w \in C^{\infty}(\bar{\Omega})$ and $H_{0} \in C^{\infty}\left(\mathbb{S}^{n-1}\right)$.

Let $u$ be a solution to the Neumann problem

$$
\begin{cases}w^{-1} \operatorname{div}(w \nabla u)=b_{\Omega} & \text { in } \Omega, \\ \frac{\partial u}{\partial v}=H_{0}(v) & \text { on } \partial \Omega,\end{cases}
$$

where $b_{\Omega} \in \mathbb{R}$ is chosen so that the problem has a unique solution up to an additive constant, that is,

$$
b_{\Omega}=P_{w, H_{0}}(\Omega ; \Sigma) / w(\Omega) .
$$

Since $w$ is positive and smooth in $\bar{\Omega}$, and $H_{0}, v$, and $\Omega$ are smooth, we deduce that $u \in C^{\infty}(\bar{\Omega})$. For these classical facts (including the existence part given by the Fredholm alternative), see [32, Example 2 in Section 10.5] or [30, end of Section 6.7].

Consider the lower contact set of $u$, defined by

$$
\Gamma_{u}=\{x \in \Omega: u(y) \geq u(x)+\nabla u(x) \cdot(y-x) \text { for all } y \in \bar{\Omega}\} .
$$

We claim that

$$
W_{0} \subset \nabla u\left(\Gamma_{u}\right) \cap \Sigma .
$$


To prove (5.6), we proceed as in the proof of Theorem 1.2 in Section 4. Take $p \in W_{0}$, that is, $p \in \mathbb{R}^{n}$ satisfying $p \cdot v<H_{0}(v)$ for each $v \in \mathbb{S}^{n-1}$. Let $x \in \bar{\Omega}$ be such that

$$
\min _{y \in \bar{\Omega}}\{u(y)-p \cdot y\}=u(x)-p \cdot x .
$$

If $x \in \partial \Omega$ then the exterior normal derivative of $u(y)-p \cdot y$ at $x$ would be nonpositive, and hence $(\partial u / \partial v)(x) \leq p \cdot v<H_{0}(p)$, contradicting (5.4). Thus, $x \in \Omega, p=\nabla u(x)$, and $x \in \Gamma_{u}$-see Section 4 for more details. Hence, $W_{0} \subset \nabla u\left(\Gamma_{u}\right)$, and since $W_{0} \subset \Sigma$, claim (5.6) follows.

Therefore,

$$
w\left(W_{0}\right) \leq \int_{\nabla u\left(\Gamma_{u}\right) \cap \Sigma} w(p) d p \leq \int_{\Gamma_{u} \cap(\nabla u)^{-1}(\Sigma)} w(\nabla u) \operatorname{det} D^{2} u d x .
$$

We have applied the area formula to the smooth map $\nabla u: \Gamma_{u} \rightarrow \mathbb{R}^{n}$, and we have used the fact that its Jacobian, det $D^{2} u$, is nonnegative in $\Gamma_{u}$ by definition of this set.

Using the arithmetic-geometric mean inequality as in Section 4 , we find that

$$
\operatorname{det} D^{2} u \leq\left(\frac{\Delta u}{n}\right)^{n} \text {. }
$$

Furthermore, when $\alpha>0$, we have the inequality

$$
s^{\alpha} t^{n} \leq\left(\frac{\alpha s+n t}{\alpha+n}\right)^{\alpha+n} \quad \text { for all } s>0 \text { and } t>0,
$$

which follows from the concavity of the logarithm function. Using also Lemma 5.1, we find

$$
\frac{w(\nabla u)}{w(x)}\left(\frac{\Delta u}{n}\right)^{n} \leq\left(\frac{\alpha\left(\frac{w(\nabla u)}{w(x)}\right)^{1 / \alpha}+\Delta u}{\alpha+n}\right)^{\alpha+n} \leq\left(\frac{\frac{\nabla w(x) \cdot \nabla u}{w(x)}+\Delta u}{D}\right)^{D} .
$$

Recall that $D=n+\alpha$. Thus, combining the previous inequalities we get

$$
\frac{w(\nabla u)}{w(x)} \operatorname{det} D^{2} u \leq\left(\frac{\frac{\nabla w(x) \cdot \nabla u}{w(x)}+\Delta u}{\alpha+n}\right)^{\alpha+n} .
$$

If $\alpha=0$ then $w \equiv 1$, and (5.8) also holds.

Now, the equation in (5.4) gives

$$
\frac{\nabla w(x) \cdot \nabla u}{w(x)}+\Delta u=\frac{\operatorname{div}(w(x) \nabla u)}{w(x)} \equiv b_{\Omega},
$$

and thus using (5.5) we find

$$
\begin{aligned}
\int_{\Gamma_{u} \cap(\nabla u)^{-1}(\Sigma)} w(\nabla u) \operatorname{det} D^{2} u d x & \leq \int_{\Gamma_{u} \cap(\nabla u)^{-1}(\Sigma)} w(x)\left(\frac{b_{\Omega}}{D}\right)^{D} d x \\
\leq & \int_{\Gamma_{u}} w(x)\left(\frac{b_{\Omega}}{D}\right)^{D} d x=\left(\frac{P_{w, H_{0}}(\Omega ; \Sigma)}{D w(\Omega)}\right)^{D} w\left(\Gamma_{u}\right) .
\end{aligned}
$$


Therefore, from (5.7) and (5.9) we deduce

$$
w\left(W_{0}\right) \leq\left(\frac{P_{w, H_{0}}(\Omega ; \Sigma)}{D w(\Omega)}\right)^{D} w\left(\Gamma_{u}\right) \leq\left(\frac{P_{w, H_{0}}(\Omega ; \Sigma)}{D w(\Omega)}\right)^{D} w(\Omega) .
$$

Finally, since by (1.14), we have $P_{w, H_{0}}(W ; \Sigma)=D w\left(W_{0}\right)$, we deduce (5.3).

An alternative way to see that (5.10) is equivalent to (5.3) (we find this way instructive in order to understand why our proof works) is to analyze the previous argument when $\Omega=W_{0}=W \cap \Sigma$. In this case $\bar{\Omega} \nsubseteq \Sigma$, and therefore, as explained in Section 3, we must solve the problem

$$
\begin{cases}w^{-1} \operatorname{div}(w \nabla u)=b_{\Omega} & \text { in } \Omega, \\ \partial u / \partial v=H_{0}(v) & \text { on } \partial \Omega \cap \Sigma, \\ \partial u / \partial v=0 & \text { on } \partial \Omega \cap \partial \Sigma,\end{cases}
$$

instead of problem (5.4). When $\Omega=W_{0}$, the solution to problem (5.11) is

$$
u(x)=|x|^{2} / 2 \text {. }
$$

For this function $u$ we have $\Gamma_{u}=W_{0}$ and $b_{W_{0}}=P_{w, H_{0}}\left(W_{0} ; \Sigma\right) / w\left(W_{0}\right)$-as in (5.5). Hence, for these concrete $\Omega$ and $u$ one verifies that all inclusions and inequalities in (5.6)-(5.10) are equalities, and thus (5.3) follows.

Case 2: $E=U \cap \Sigma$, where $U$ is a bounded smooth open set in $\mathbb{R}^{n}$. Even though both $U$ and $\Sigma$ are Lipschitz sets, their intersection might not be Lipschitz (for instance if $\partial U$ and $\partial \Sigma$ meet tangentially at a point). As a consequence, approximating $U \cap \Sigma$ by smooth sets converging in perimeter is a more subtle issue. However, we claim that there exists a sequence $\left\{\Omega_{k}\right\}_{k \geq 1}$ of smooth bounded domains satisfying

$$
\overline{\Omega_{k}} \subset \Sigma \text { and } \quad \lim _{k \rightarrow \infty} \frac{P_{w, H_{0}}\left(\Omega_{k} ; \Sigma\right)}{w\left(\Omega_{k}\right)^{(D-1) / D}} \leq \frac{P_{w, H_{0}}(E ; \Sigma)}{w(E)^{(D-1) / D}} .
$$

Case 2 follows immediately using this claim and what we have proved in Case 1 . We now proceed to prove the claim.

It is no restriction to assume that $e_{n}$, the $n$th vector of the standard basis, belongs to the cone $\Sigma$. Then, $\partial \Sigma$ is a convex graph (and therefore Lipschitz in every compact set) over the variables $x_{1}, \ldots, x_{n-1}$. That is,

$$
\Sigma=\left\{x_{n}>g\left(x_{1}, \ldots, x_{n-1}\right)\right\}
$$

for some convex function $g: \mathbb{R}^{n-1} \rightarrow \mathbb{R}$.

First we construct a sequence

$$
F_{k}=\left\{x_{n}>g_{k}\left(x_{1}, \ldots, x_{n-1}\right)\right\}, \quad k \geq 1,
$$

of convex smooth sets whose boundary is a graph $g_{k}: \mathbb{R}^{n-1} \rightarrow \mathbb{R}$ over the first $n-1$ variables and satisfying:

(i) $g_{1}>g_{2}>\cdots$ in $\bar{B}$, where $B$ is a large ball $B \subset \mathbb{R}^{n-1}$ containing the projection of $\bar{U}$.

(ii) $g_{k} \rightarrow g$ uniformly in $\bar{B}$. 
(iii) $\nabla g_{k} \rightarrow \nabla g$ almost everywhere in $\bar{B}$ and $\left|\nabla g_{k}\right|$ is bounded independently of $k$.

(iv) The smooth manifolds $\partial F_{k}=\left\{x_{n}=g_{k}\left(x_{1}, \ldots, x_{n-1}\right)\right\}$ and $\partial U$ intersect transversally.

To construct the sequence $g_{k}$, we consider the convolution of $g$ with a standard mollifier $\eta \in C_{c}^{\infty}\left(B_{1}\right)$,

$$
\tilde{g}_{k}=g * k^{n-1} \eta(k x)+C / k,
$$

where $C$ is a large constant (depending on $\|\nabla g\|_{L^{\infty}\left(\mathbb{R}^{n-1}\right)}$ ) to guarantee $\tilde{g}_{k}>g$ in $\bar{B}$. Note that the existence of $C$ follows from the inequality

$$
g * k^{n-1} \eta(k x) \geq \inf _{B_{1 / k}(x)} g \geq g(x)-\frac{1}{k}\|\nabla g\|_{L^{\infty}} .
$$

It follows that a subsequence of $\tilde{g}_{k}$ will satisfy (i)-(iii). Next, by a version of Sard's Theorem [31, Section 2.3] almost every small translation of the smooth manifold $\left\{x_{n}=\right.$ $\left.\tilde{g}_{k}\left(x_{1}, \ldots, x_{n-1}\right)\right\}$ will intersect $\partial U$ transversally. Thus, the sequence

$$
g_{k}\left(x_{1}, \ldots, x_{n-1}\right)=\tilde{g}_{k}\left(x_{1}-y_{1}^{k}, \ldots, x_{n-1}-y_{n-1}^{k}\right)+y_{n}^{k}
$$

will satisfy (i)-(iv) if $y^{k} \in \mathbb{R}^{n}$ are chosen with $\left|y^{k}\right|$ sufficiently small depending on $k$-in particular to preserve (i).

Let us show now that $P_{w, H_{0}}\left(U \cap F_{k} ; \Sigma\right)$ converges to $P_{w, H_{0}}(E ; \Sigma)$ as $k \uparrow \infty$. Note that (i) yields $F_{k} \subset F_{k+1}$ for all $k \geq 1$. This monotonicity will be useful to prove the convergence of perimeters, which we do next.

Indeed, since we have considered the gauge $H_{0}$ instead of $H$, we have

$$
P_{w, H_{0}}(E ; \Sigma)=\int_{\partial U \cap \Sigma} H_{0}(v(x)) w(x) d x=\int_{\partial E} H_{0}(v(x)) w(x) d x .
$$

This is because $\partial E=\partial(U \cap E) \subset(\partial U \cap \Sigma) \cup(\bar{U} \cap \partial \Sigma)$ and

$$
H_{0}(v(x))=0 \quad \text { for almost all } x \in \partial \Sigma .
$$

Now, since $\partial\left(U \cap F_{k}\right) \subset\left(\partial U \cap F_{k}\right) \cup\left(\bar{U} \cap \partial F_{k}\right)$, we have

$$
0 \leq P_{w, H_{0}}\left(U \cap F_{k} ; \Sigma\right)-\int_{\partial U \cap F_{k}} H_{0}(v(x)) w(x) d x \leq \int_{\bar{U} \cap \partial F_{k}} H_{0}\left(v_{F_{k}}(x)\right) w(x) d x .
$$

On the one hand, using dominated convergence, (5.13), (5.14), (ii)-(iii), and (5.16), we readily prove that

$$
\int_{\bar{U} \cap \partial F_{k}} H_{0}\left(v_{F_{k}}(x)\right) w(x) d x \rightarrow 0 .
$$

On the other hand, by (i) and (ii), $F_{k} \cap(B \times \mathbb{R})$ is an increasing sequence exhausting $\Sigma \cap(B \times \mathbb{R})$. Hence, by monotone convergence,

$$
\int_{\partial U \cap F_{k}} H_{0}(v(x)) w(x) d x \rightarrow \int_{\partial U \cap \Sigma} H_{0}(v(x)) w(x) d x=P_{w, H_{0}}(E ; \Sigma) .
$$


Therefore, the sets $U \cap F_{k}$ approximate $U \cap \Sigma$ in $L^{1}$ and in $\left(w, H_{0}\right)$-perimeter. Moreover, by (iv), $U \cap F_{k}$ are Lipschitz open sets.

Finally, to obtain the sequence of smooth domains $\Omega_{k}$ in (5.12), we use a partition of unity and local regularization of the Lipschitz sets $U \cap F_{k}$ to guarantee the convergence of the $\left(w, H_{0}\right)$-perimeters. In case the regularized sets had more than one connected component, we may always choose the one having the best isoperimetric quotient.

Case 3: $E$ is any measurable set with $w(E)<\infty$ and $P_{w, H_{0}}(E ; \Sigma) \leq P_{w, H}(E ; \Sigma)<\infty$. As a consequence of [2, Theorem 5.1], $C_{c}^{\infty}\left(\mathbb{R}^{n}\right)$ is dense in the space $\mathrm{BV}_{\mu, H_{0}}$ of functions of bounded variation with respect to the measure $\mu=w \chi_{\Sigma}$ and the gauge $H_{0}$. Note that our definition of $P_{w, H_{0}}(E ; \Sigma)$ coincides with the $\left(\mu, H_{0}\right)$-total variation of the characteristic function $\chi_{E}$, that is, $\left|D_{\mu} \chi_{E}\right|_{H_{0}}$ in the notation of [2]. Hence, by the coarea formula in [2, Theorem 4.1] and the argument in [37, Section 6.1.3], we find that for each measurable set $E \subset \Sigma$ with finite measure there exists a sequence of bounded smooth sets $\left\{U_{k}\right\}$ satisfying

$$
\lim _{k \rightarrow \infty} w\left(U_{k} \cap \Sigma\right)=w(E) \quad \text { and } \quad \lim _{k \rightarrow \infty} P_{w, H_{0}}\left(U_{k} ; \Sigma\right)=P_{w, H_{0}}(E ; \Sigma) .
$$

Then we are back to Case 2 above, and hence the proof is finished.

The previous proof is simpler when $w$ vanishes on $\partial \Sigma$, as explained in the following remark.

Remark 5.2. In the proof of Theorem 1.3, Cases 2 and 3 are deduced from Case 1 (the inequality for smooth domains at positive distance from $\partial \Sigma$ ) by approximation arguments. To correctly approximate the relative perimeter we need to know that, for any given sequence of pieces of surface converging (in $C^{1}$ norm, say) to $\partial \Sigma$, their weighted anisotropic perimeter necessarily converges to zero. This is why we define the auxiliary gauge $H_{0}$, which vanishes on directions normal to $\partial \Sigma$.

In the case $w=0$ on $\partial \Sigma$ there is no need to consider this auxiliary gauge. Indeed, since $w(x) \rightarrow 0$ as $x \rightarrow \partial \Sigma$, the weighted perimeters of pieces of surfaces approximating $\partial \Sigma$ (even only in $C^{0}$ norm) will converge to zero. This makes the proof and the approximation arguments simpler in this case. In particular, when $H=\|\cdot\|_{2}$ and $w=0$ on $\partial \Sigma$, the proof of the weighted isoperimetric inequality does not involve auxiliary anisotropic perimeters. Indeed, in this case we can prove the weighted isoperimetric inequality by solving the simpler Neumann problem

$$
\begin{cases}w^{-1} \operatorname{div}(w \nabla u)=b_{\Omega} & \text { in } \Omega, \\ \partial u / \partial v=1 & \text { on } \partial \Omega .\end{cases}
$$

After the announcement of our result and proof in [10], Emanuel Milman showed us a nice geometric construction that yields the weighted inequality in Theorem 1.3 when $\alpha$ is a nonnegative integer. We next sketch his construction.

Remark 5.3 (Emanuel Milman's construction). When $\alpha$ is a nonnegative integer, the weighted isoperimetric inequality of Theorem 1.3 (with $H=\|\cdot\|_{2}$ ) can be proved as a 
limit case of the Lions-Pacella inequality in convex cones of $\mathbb{R}^{n+\alpha}$. Indeed, let $w^{1 / \alpha}>0$ be a concave function, homogeneous of degree 1 , in an open convex cone $\Sigma \subset \mathbb{R}^{n}$. For each $\varepsilon>0$, consider the cone

$$
\mathcal{C}_{\varepsilon}=\left\{(x, y) \in \mathbb{R}^{n} \times \mathbb{R}^{\alpha}: x \in \Sigma,|y|<\varepsilon w(x)^{1 / \alpha}\right\} .
$$

From the convexity of $\Sigma$ and the concavity of $w^{1 / \alpha}$ we find that $\mathcal{C}_{\varepsilon}$ is a convex cone. Hence, by Theorem 1.1,

$$
\frac{P\left(\tilde{E} ; \mathcal{C}_{\varepsilon}\right)}{\left|\tilde{E} \cap \mathcal{C}_{\varepsilon}\right|^{\frac{n+\alpha-1}{n+\alpha}}} \geq \frac{P\left(\mathcal{B}_{1} ; \mathcal{C}_{\varepsilon}\right)}{\left|\mathcal{B}_{1} \cap \mathcal{C}_{\varepsilon}\right|^{\frac{n+\alpha-1}{n+\alpha}}} \quad \text { for all } \tilde{E} \text { with }\left|\tilde{E} \cap \mathcal{C}_{\varepsilon}\right|<\infty
$$

where $\mathcal{B}_{1}$ is the unit ball of $\mathbb{R}^{n+\alpha}$. Now, given a Lipschitz set $E \subset \mathbb{R}^{n}$, by considering the cylinder $\tilde{E}=E \times \mathbb{R}^{\alpha}$ one finds

$$
\left|\tilde{E} \cap \mathcal{C}_{\varepsilon}\right|=\int_{E \cap \Sigma} d x \int_{\left\{|y|<\varepsilon w(x)^{1 / \alpha}\right\}} d y=\omega_{\alpha} \varepsilon^{\alpha} \int_{E \cap \Sigma} w(x) d x=\omega_{\alpha} \varepsilon^{\alpha} w(E \cap \Sigma)
$$

and

$$
P\left(\tilde{E} ; \mathcal{C}_{\varepsilon}\right)=\int_{\partial E \cap \Sigma} d S(x) \int_{\left\{|y|<\varepsilon w(x)^{1 / \alpha}\right\}} d y=\omega_{\alpha} \varepsilon^{\alpha} \int_{\partial E \cap \Sigma} w(x) d S=\omega_{\alpha} \varepsilon^{\alpha} P_{w}(E ; \Sigma) .
$$

On the other hand, one easily sees that, as $\varepsilon \downarrow 0$,

$$
\frac{P\left(\mathcal{B}_{1} ; \mathcal{C}_{\varepsilon}\right)}{\left|\mathcal{B}_{1} \cap \mathcal{C}_{\varepsilon}\right|^{\frac{n+\alpha-1}{n+\alpha}}}=\left(\omega_{\alpha} \varepsilon^{\alpha}\right)^{\frac{1}{n+\alpha}}\left(\frac{P_{w}\left(B_{1} ; \Sigma\right)}{w\left(B_{1} \cap \Sigma\right)^{\frac{n+\alpha-1}{n+\alpha}}}+o(1)\right)
$$

where $B_{1}$ is the unit ball of $\mathbb{R}^{n}$. Hence, letting $\varepsilon \downarrow 0$ in (5.17) one obtains

$$
\frac{P_{w}(E ; \Sigma)}{w(E \cap \Sigma)^{\frac{n+\alpha-1}{n+\alpha}}} \geq \frac{P_{w}\left(B_{1} ; \Sigma\right)}{w\left(B_{1} \cap \Sigma\right)^{\frac{n+\alpha-1}{n+\alpha}}}
$$

which is the inequality of Theorem 1.3 in the case $H=\|\cdot\|_{2}$ and $\alpha$ is an integer.

Acknowledgments. The authors were supported by grants MINECO MTM2011-27739-C04-01 and MTM2014-52402-C3-1-P. They are part of the Catalan research group 2014 SGR 1083

\section{References}

[1] Bauschke, H. H., Güler, O., Lewis, A. S., Sendov, H. S.: Hyperbolic polynomials and convex analysis. Canad. J. Math. 53, 470-488 (2001) Zbl 0974.90015 MR 1827817

[2] Bellettini, G., Bouchitté, G., Fragalà, I.: BV functions with respect to a measure and relaxation of metric integral functionals. J. Convex Anal. 6, 349-366 (1999) Zbl 0959.49015 MR 1736243

[3] Borell, C.: The Brunn-Minkowski inequality in Gauss space. Invent. Math. 30, 207-216 (1975) Zbl 0292.60004 MR 0399402 
[4] Brock, F., Chiacchio, F., Mercaldo, A.: Weighted isoperimetric inequalities in cones and applications. Nonlinear Anal. 75, 5737-5755 (2012) Z Zbl 1253.26049 MR 2948294

[5] Brock, F., Chiacchio, F., Mercaldo, A.: A weighted isoperimetric inequality in an orthant. Potential Anal. 41, 171-186 (2014) Zbl 1303.26024 MR 3225812

[6] Cabré, X.: Partial differential equations, geometry, and stochastic control, Butl. Soc. Catalana Mat. 15, 7-27 (2000) (in Catalan) MR 1796275

[7] Cabré, X.: Elliptic PDE's in probability and Geometry: symmetry and regularity of solutions. Discrete Contin. Dynam. System. 20, 425-457 (2008) Zbl 1158.35033 MR 2373200

[8] Cabré, X., Ros-Oton, X.: Regularity of stable solutions up to dimension 7 in domains of double revolution. Comm. Partial Differential Equations 38, 135-154 (2013) Zbl 1268.35059 MR 3005549

[9] Cabré, X., Ros-Oton, X.: Sobolev and isoperimetric inequalities with monomial weights. J. Differential Equations 255, 4312-4336 (2013) Zbl 1293.46018 MR 3097258

[10] Cabré, X., Ros-Oton, X., Serra, J.: Euclidean balls solve some isoperimetric problems with nonradial weights. C. R. Math. Acad. Sci. Paris 350, 945-947 (2012) Zbl 1258.52001 MR 2996771

[11] Caffarelli, L. A., Nirenberg, L., Spruck, J.: The Dirichlet problem for nonlinear second order elliptic equations, III: Functions of the eigenvalues of the Hessian. Acta Math. 155, 261-301 (1985) Zbl 0654.35031 MR 0806416

[12] Cañete, A., Rosales, C.: Compact stable hypersurfaces with free boundary in convex solid cones with homogeneous densities. Calc. Var. Partial Differential Equations 51, 887-913 (2014) Zbl 1317.53006 MR 3268875

[13] Carroll, C., Jacob, A., Quinn, C., Walters, R.: The isoperimetric problem on planes with density. Bull. Austral. Math. Soc. 78, 177-197 (2008) Zbl 1161.53049 MR 2466858

[14] Chambers, G.: Proof of the log-convex density conjecture. J. Eur. Math. Soc., to appear

[15] Chavel, I.: Riemannian Geometry: A Modern Introduction. 2nd rev. ed., Cambridge Univ. Press, Cambridge (2006) Zbl 1099.53001 MR 2229062

[16] Cianchi, A., Fusco, N., Maggi, F., Pratelli, A.: On the isoperimetric deficit in the Gauss space. Amer. J. Math. 133, 131-186 (2011) Zbl 1219.28005 MR 2752937

[17] Cinti, E., Pratelli, A.: The $\varepsilon-\varepsilon^{\beta}$ property, the boundedness of isoperimetric sets in $\mathbb{R}^{n}$ with density, and some applications. J. Reine Angew. Math., DOI: 10.1515/crelle-2014-0120 (2015)

[18] Cordero-Erausquin, D., Nazaret, B., Villani, C.: A mass transportation approach to sharp Sobolev and Gagliardo-Nirenberg inequalities. Adv. Math. 182, 307-332 (2004) Zbl 1048.26010 MR 2032031

[19] Dahlberg, J., Dubbs, A., Newkirk, E., Tran, H.: Isoperimetric regions in the plane with density $r^{p}$. New York J. Math. 16, 31-51 (2010) Zbl 1196.53008 MR 2645984

[20] Díaz, A., Harman, N., Howe, S., Thompson, D.: Isoperimetric problem in sectors with density. Adv. Geom. 14, 589-619 (2012) Zbl 1263.49061 MR 3005102

[21] Dinghas, A.: Über einen geometrischen Satz von Wulff für die Gleichgewichtsform von Kristallen. Zeitschrift für Kristallographie 105, 304-314 (1944) Zbl 0028.43001 MR 0012454

[22] Figalli, A., Indrei, E.: A sharp stability result for the relative isoperimetric inequality inside convex cones. J. Geom. Anal. 23, 938-969 (2013) Zbl 1262.49045 MR 3023863

[23] Figalli, A., Maggi, F.: On the isoperimetric problem for radial log-convex densities. Calc. Var. Partial Differential Equations 48, 447-489 (2013) Zbl 1307.49046 MR 3116018

[24] Figalli, A., Maggi, F., Pratelli, A.: A mass transportation approach to quantitative isoperimetric inequalities. Invent. Math. 182, 167-211 (2010) Zbl 1196.49033 MR 2672283 
[25] Fonseca, I., Müller, S.: A uniqueness proof for the Wulff theorem. Proc. Roy. Soc. Edinburgh Sect. A 119, 125-136 (1991) Zbl 0725.49017 MR 1130601

[26] Fusco, N., Maggi, F., Pratelli, A.: On the isoperimetric problem with respect to a mixed Euclidean-Gaussian density. J. Funct. Anal. 260, 3678-3717 (2011) Zbl 1222.49058 MR 2781973

[27] Gårding, L.: Linear hyperbolic differential equations with constant coefficients. Acta Math. 85, 1-62 (1951) Zbl 0045.20202 MR 0041336

[28] Gårding, L.: An inequality for hyperbolic polynomials. Indiana Univ. Math. J. 8, 957-965 (1959) Zbl 0090.01603 MR 0113978

[29] Gardner, R. J.: The Brunn-Minkowski inequality. Bull. Amer. Math. Soc. 39, 355-405 (2002) Zbl 1019.26008 MR 1898210

[30] Gilbarg, D., Trudinger, N. S.: Elliptic Partial Differential Equations of Second Order. 2nd ed., Springer, Berlin (1983) Zbl 0562.35001 MR 0737190

[31] Guillemin, V., Pollack, A.: Differential Topology. AMS Chelsea (2010) Zbl 05788282 MR 2680546

[32] Hörmander, L.: Linear Partial Differential Operators. Springer (1969) Zbl 0175.39201 MR 0248435

[33] Lions, P.-L., Pacella, F.: Isoperimetric inequality for convex cones. Proc. Amer. Math. Soc. 109, 477-485 (1990) Zbl 0717.52008 MR 1000160

[34] Maderna, C., Salsa, S.: Sharp estimates for solutions to a certain type of singular elliptic boundary value problems in two dimensions. Applicable Anal. 12, 307-321 (1981) Zbl 0445.35016 MR 0653203

[35] Marcus, M., Lopes, L.: Inequalities for symmetric functions and hermitian matrices. Canad. J. Math. 9, 305-312 (1957) Zbl 0079.02103 MR 0084541

[36] Maurmann, Q., Morgan, F.: Isoperimetric comparison theorems for manifolds with density. Calc. Var. Partial Differential Equations 36, 1-5 (2009) Zbl 1175.49042 MR 2507612

[37] Maz’ja, V. G.: Sobolev Spaces. Springer, Berlin (1985) Zbl 0692.46023 MR 0817985

[38] McCann, R. J.: Equilibrium shapes for planar crystals in an external field. Comm. Math. Phys. 195, 699-723 (1998) Zbl 0936.74029 MR 1641031

[39] Milman, E., Rotem, L.: Complemented Brunn-Minkowski inequalities and isoperimetry for homogeneous and non-homogeneous measures. Adv. Math. 262, 867-908 (2014) Zbl 1311.52008 MR 3228444

[40] Morgan, F.: Riemannian Geometry: A Beginner's Guide. Jones and Bartlett (1993) Zbl 0794.53001 MR 1174293

[41] Morgan, F.: Manifolds with density. Notices Amer. Math. Soc. 52, 853-858 (2005) Zbl 1118.53022

[42] Morgan, F.: The log-convex density conjecture. Frank Morgan's blog (April 2010), http://sites.williams.edu/Morgan/2010/04/03/the-log-convex-density-conjecture/

[43] Morgan, F., Pratelli, A.: Existence of isoperimetric regions in $\mathbb{R}^{n}$ with a density. Ann. Global Anal. Geom. 43, 331-365 (2013) Zbl 1263.49049 MR 3038539

[44] Nardi, G.: Schauder estimation for solutions of Poisson's equation with Neumann boundary condition. Enseign. Math. 60, 421-437 (2014) Zbl 1317.35047 MR 3342652

[45] Ritoré, M., Rosales, C.: Existence and characterization of regions minimizing perimeter under a volume constraint inside Euclidean cones. Trans. Amer. Math. Soc. 356, 4601-4622 (2004) Zbl 1057.53023 MR 2067135

[46] Rosales, C., Cañete, A., Bayle, V., Morgan, F.: On the isoperimetric problem in Euclidean space with density. Calc. Var. Partial Differential Equations 31, $27-46$ (2008) Zbl 1126.49038 MR 2342613 
[47] Talenti, G.: A weighted version of a rearrangement inequality. Ann. Univ. Ferrara 43, 121-133 (1997) Zbl 0936.26007 MR 1686750

[48] Taylor, J.: Existence and structure of solutions to a class of nonelliptic variational problems. In: Symposia Math. XIV (Roma, 1973), Academic Press, 499-508 (1974) Zbl 0317.49053 MR 0420407

[49] Taylor, J.: Unique structure of solutions to a class of nonelliptic variational problems. In: Differential Geometry (Stanford, CA, 1973), Proc. Sympos. Pure Math. 27, Amer. Math. Soc., 419-427 (1975) Zbl 0317.49054 MR 0388225

[50] Taylor, J.: Crystalline variational problems. Bull. Amer. Math. Soc. 84, 568-588 (1978) Zbl 0392.49022 MR 0493671

[51] Trudinger, N. S.: Isoperimetric inequalities for quermassintegrals. Ann. Inst. H. Poincaré Anal. Non Linéaire 11, 411-425 (1994) Zbl 0859.52001 MR 1287239

[52] Villani, C.: Optimal Transport, Old And New. Grundlehren Math. Wiss. 338, Springer (2009) Zbl 1156.53003 MR 2459454

[53] Wulff, G.: Zur Frage der Geschwindigkeit des Wachstums und der Auflösung der Kristallflächen. Zeitschrift für Kristallographie 34, 449-530 (1901) 